\title{
Continu d'émission UV-VUV du néon dans des plasmas à haute pression
}

\author{
J. M. Pouvesle et C. Cachoncinlle \\ GREMI, CNRS, Université d'Orléans, URA 831 UFR SFA, BP. 6759, 45067 Orléans Cedex \\ 02, France
}

\begin{abstract}
Résumé: L'utilisation de décharges controlées par diélectrique est un moyen efficace pour étudier la fluorescence UV-VUV de gaz ou mélanges de gaz à haute pression. Nous rapportons ici la mise en évidence d'un continu intense du Néon s'étendant de 160 à plus de $400 \mathrm{~nm}$ et présentant un maximum autour de $240 \mathrm{~nm}$.

Abstract: The use of dielectric controlled discharges is a convenient means to study the UV-VUV fluorescence of high pressure pure gas or gas mixtures. We report in this work the observation of a strong emission continuum in Neon extending from 160 to more than $400 \mathrm{~nm}$ and peaking around $240 \mathrm{~nm}$.
\end{abstract}

L'utilisation de nouvelles techniques expérimentales pour la production de fluorescences dans le dommaine UV-VUV a contribué à relancer les études expérimentales et théoriques sur les gaz rares purs et mélanges à base de gaz rares. Depuis les travaux de Tanaka sur les continus vUV des gaz rares [1], il a été mis en évidence dans de très nombreuses expériences d'autres continus de fluorescence à de plus grandes longueurs d'ondes s'étendant dans certains cas jusqu'au proche ultra-violet. Les états à l'origine de ces émissions, d'une largeur spectrale souvent importante comparée au deux premiers continus VUV, ne sont pas encore tout à fait clairement identifiés. Cependant, leur attribution à des transitions faisant intervenir des ions moléculaires semble s'imposer $[2,3]$.

Dans le cadre d'un travail plus général sur les continus des gaz rares, nous rapportons ici l'observation (voir figure 1) d'un continu intense de fluorescence UV-VUV dans un plasma de néon pur excité en décharge controlée par diélectrique à des pressions comprises entre 1 et 5 bars. A notre connaissance cette fluorescence n'avait encore jamais été mise en évidence dans des conditions de décharge.

Notre système est caractérisé par une très haute tension $(15-60 \mathrm{kV})$ appliquée aux bornes des électrodes isolées du gaz par une barrière diélectrique et un temps d'excitation très court (10 ns). Le débit du gaz Ne (pureté 99.99\%) à l'intérieur de la cellule $\left(7 \mathrm{~cm}^{3}\right)$ est compris entre 1 et $2 \mathrm{l} / \mathrm{min}$. Le taux de répétition est fixé à $20 \mathrm{~Hz}$. L'analyse de la fluorescence est réalisée à travers une fenêtre en $\mathrm{MgF2}$ au moyen d'un spectromètre UV-VUV de faible résolution couplé à un échantillonneur rapide (Tektronix 2440) permettant des études de spectroscopie résolue en temps. Les spectres obtenus ne sont pas corrigés de la réponse spectrale du système (spectromètre H20 UVL Jobin \& Yvon, PM Hammamatsu R1220 et R955). 


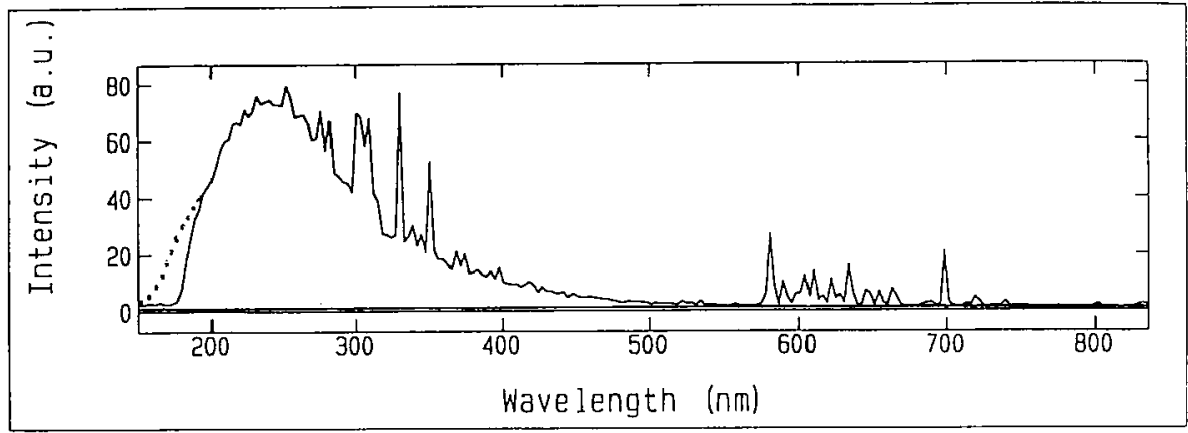

Figure 1 : Continu d'émission du Néon excité par DCD; P = 5 bars, PM R955. (la courbe en pointillés correspond à l'engistrement dans les mêmes conditions expérimentales avec un PM R1220 et sans la coupure de l'air).

Dans ces conditions expérimentales le plasma de néon émet un continu s'étendant de $160 \mathrm{~nm}$ à plus de $400 \mathrm{~nm}$. Cette émission est très fortement dépendante de la pression; son intensité croit pratiquement comme $\mathrm{P}^{2}$ (figure 2 ). L'analyse temporelle de ce continu montre une durée de vie typique d'une centaine de nanosecondes dans ce domaine de pression.

Comme dans le cas de l'argon [3,4], l'analyse des résultats expérimentaux nous permet de proposer 1'intervention des états de Rydperg de l'ion moléculaire $\mathrm{Ne}^{+*}$ pour expliquer le très large continu de fluorescence observé. On peut en effet s'attendre à ce que les états ioniques de Rydberg de tous les gaz rares présentent des puits de potentiel à très courtes distances internucléaires comme cela a déjà été établi pour He et Ar. Le schéma cinétique de peuplement pourrait faire intervenir la réaction suivante:

$$
\mathrm{Ne}^{+}+\mathrm{Ne}^{*}+\mathrm{Ne} \rightarrow \mathrm{Ne}_{2}^{+*}+\mathrm{Ne}
$$

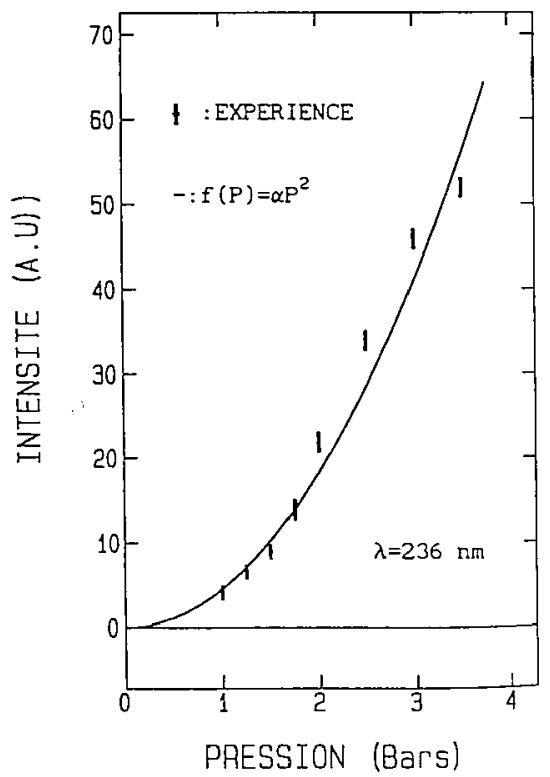

Figure 2 : Dépendence en fonction de la pression de Néon de I'intensité de fluorescence à 236 nanomètres.

[1] TANAKA Y., JURSA A.S., and LEBLANC F.J., J. OPT. SOC. AM. 48 (1958) 304

[2] LANGHOFF H., OPTICS COMM. 61 (1988) 31

[3] CACHONCINLLE C., POUVESLE J.M., DAVANLOO F., and COLLiNS C.B., Optics Comm. 79 (1990) 41

[4] CACHONCINLlE C., POUVESLE J.M., DURAND G., SPIEGELMANN F., J. CHEM. PHYS. 96 (1992) 6085, J. CHEM. PHYS. 96 (1992) 6093 